\title{
The Identification of Migraine Using Functional Connectivity Pattern of Multi-Networks by Deep Learning with Genetic Optimization
}

\author{
Yuhu Shi \\ Information Engineering College, Shanghai Maritime University, Shanghai, China \\ syhustb2011@163.com
}

Keywords: fMRI, Group independent component analysis, Back-propagation, Genetic algorithm, Migraine.

\begin{abstract}
Migraine is a kind of chronic functional disorder characterized by recurrent pain, and its pathogenesis is still unclear. Therefore, researchers have conducted a large number of studies on migraine by using functional magnetic resonances imagine (fMRI) technology in recent years. On this basis, we explored the performance of functional connectivities (FCs) between resting-state brain functional networks for the identification of migraine in this paper. Specifically, eight resting-state brain functional networks were obtained from fMRI data of 34 migraine patients and 40 healthy control subjects by group independent component analysis with spatiotemporal dual-regression. Then, the FCs between these brain networks were used as the features for the classification of migraine patients and healthy subjects by back-propagation and genetic algorithms, and good prediction performance was obtained in predicting migraine, which means these resting-state networks and the FCs between them played an key role in the brain functional activity of migraine. Therefore, it may be provided a new perspective for the clinical diagnosis of migraine.
\end{abstract}

\section{Introduction}

Migraine is a complex and subjective experience that includes sensory-discriminative, affective and cognitive aspects. Neuroimaging studies have shown functional alterations in widespread brain regions of migraine brain, which involved in pain processing [1-3]. Resting-state functional magnetic resonance imaging (rsfMRI) has been useful for delineating the neural underpinnings of the pain experience in migraine patients. An increasing body of literature has shown that people with migraine have alterations in the functional connectivities (FCs) of regions which important for mediating sensory, affective, and cognitive components of pain [4-6].

In 2001, Raichle et al. found that there were multiple independent, spatially coherent resting-state functional networks in the brain, including: default mode network, sensorimotor network, dorsal attention network, executive control network, salience network, frontoparietal network, etc. [7], which have been confirmed by domestic and foreign scholars. In recent years, more and more researches reported that resting-state brain functional networks are directly related to pain processing, and found that these brain functional networks are abnormal in migraine patients [8-10]. However, few studies have been done on the differences of FCs between these resting-state brain functional networks in migraine patients and healthy subjects.

Machine-learning algorithms trained to automatically classify patient populations from healthy controls based on rsfMRI measures have shown good utility for discriminating patients with chronic pain from healthy controls [11-13]. But rsfMRI measures of FCs between multiple brain networks have not yet been used as input metrics for accurately discriminating migraine patients from healthy controls. The ability to classify individual migraine patients from healthy controls based on rsfMRI functional connectivity patterns would provide objective insight into the neural mechanism of migraine and would potentially provide an objective brain biomarker for migraine. The purpose of this study was to develop a new identification method for the classification of migraine patients from 
healthy controls based on the FCs patterns derived from multiple resting-state brain functional networks.

\section{Materials and methods}

\subsection{Data acquisition}

In this study, 34 migraine patients participated in the experiment came from the department of neurology of Shanghai Sixth People's Hospital East Affiliated to Shanghai University of Medicine \& Health Science using the SIEMENS magnetic resonance instrument (3T). All migraine patients were diagnosed with episodic or chronic migraine according to the diagnostic criteria set forth by the International Classification of Headache Disorders 3rd edition (beta version) (Headache Classification Committee of the International Headache Society, 2013). All procedures were approved by the Independent Ethics Committee of Shanghai Sixth People's Hospital East Campus and all the participants were informed consent before the data acquisition. In the process of data acquisition, all the subjects were instructed to keep awake without think anything. The images dataset was acquired using single-shot SENSE gradient an echo planar imaging (EPI) with 38 slices, providing whole-brain coverage and 160 volumes, a repetition time (TR) of 3s, and a scan resolution of $64 \times 64$. The in-plane resolution was $4 \mathrm{~mm} \times 4 \mathrm{~mm}$, and the slice thickness was $4 \mathrm{~mm}$.

The resting-state fMRI data of 40 healthy subjects were download from the public neuroimaging database (http://www.nitrc.org/projects/fcon 1000/), which was used as the healthy control (HC) group for the purpose of comparison. The image dataset was acquired using single-shot SENSE gradient echo EPI with 33 slices, providing whole-brain coverage and 225 volumes, a TR of 2 s, an TE of $30 \mathrm{~ms}$, a FA of 80 and a scan resolution of $64 \times 64$. The in-plane resolution was $3.13 \mathrm{~mm} \times 3.13 \mathrm{~mm}$, and the slice thickness was $3.6 \mathrm{~mm}$.

\subsection{Data preprocessing}

In the experiment, all of the fMRI data were preprocessed by using the DPARSF software (http://rfmri.org/DPARSF), the first 10 time points of each fMRI data were discarded, and the other preprocessing steps included slice timing, motion correction, spatial normalization and spatial smoothing. Next, the sinc interpolation method and six degrees transformation method were applied to eliminate the time offset and spatial offset, respectively. In order to further minimize artifacts, the data which detected any direction of displacement is more than $1.5 \mathrm{~mm}$ or head rotation angle is greater than 1.5 degrees would be abandoned. When the head motion correction was completed, all data were normalized with echo planar imaging template released by Montreal neurological research institute. Finally, Gaussian kernel of $6 \mathrm{~mm}$ was taken to smooth all the data in order to make increase the signal to noise ratio of the data.

\subsection{Group independent component analysis}

After preprocessing the data, the fMRI data from both healthy control and migraine patient groups were analyzed using spatial group independent component analysis (GICA) framework as implemented in the GIFT software [14, 15]. Spatial ICA de-composes the subject data into linear mixtures of spatially independent components with a unique time course. Particularly, spatial ICA was implemented using FastICA algorithm [16] in the experiment, and MDL [17] was used to estimate the number of independent components (ICs). To ensure stability of estimation, we repeated the ICA algorithm 20 times in ICASSO [18], and aggregate spatial maps were estimated as the modes of component clusters. Subject specific spatial maps (SMs) and time courses (TCs) were obtained using the spatiotemporal dual-regression back reconstruction approach implemented in GIFT software.

\subsection{Classification of back-propagation (BP) with genetic optimization}

A genetic algorithm is a method for solving optimization problems inspired by natural selection in biological evolution. Contrary to classical optimization algorithms, the genetic algorithm generates a 
population of points at each iteration, and it has been widely used in various optimization problems to find a global optimum solution for a set of predictor input variables [19]. Therefore, we use it to obtain the optimal parameters of back-propagation algorithm in this study.

The BP algorithm has been the core ingredient to most successes in deep learning, from image recognition to reinforcement learning [20-22]. The principle of BP neural network is that the gradient descent method is used to adjust the weights and thresholds, so that the mean square error value of the actual output of the network and the mean square error value of the expected output is minimal.

Assuming that denotes error function to be minimized by the learning process. Standard BP implements gradient descent on the $\varepsilon$, which can be applied in a stochastic fashion on-line or in batch form, by summing or averaging over all training examples. Here, we are omitting the tindex for simplicity, and then the standard BP learning rule is easily obtained by applying the chain rule and given by:

$$
\Delta \mathrm{w}_{\mathrm{ij}}^{\mathrm{h}}=-\eta \frac{\partial \varepsilon}{\partial \mathrm{w}_{\mathrm{ij}}^{\mathrm{h}}}=\eta \mathrm{B}_{\mathrm{i}}^{\mathrm{h}} \mathrm{O}_{\mathrm{j}}^{\mathrm{h}-1}
$$

where $\mathrm{w}_{\mathrm{ij}}^{\mathrm{h}}$ denotes the weight connecting neuron $\mathrm{j}$ in layer $\mathrm{h}-1$ to neuron $\mathrm{i}$ in layer $\mathrm{h}, \eta$ is the learning rate, $\mathrm{O}_{\mathrm{j}}^{\mathrm{h}-1}$ is the presynaptic activity, and $\mathrm{B}_{\mathrm{i}}^{\mathrm{h}}$ is the $\mathrm{BP}$ error. Using the chain rule, it is easy to see that the $\mathrm{BP}$ error satisfies the recurrence relation:

$$
\begin{aligned}
& \quad \mathrm{B}_{\mathrm{i}}^{\mathrm{h}}=\frac{\partial \varepsilon}{\partial \mathrm{S}_{\mathrm{i}}^{\mathrm{h}}}=\left(\mathrm{f}_{\mathrm{i}}^{\mathrm{h}}\right)^{\prime} \sum_{\mathrm{k}} \mathrm{B}_{\mathrm{k}}^{\mathrm{h}+1} \mathrm{~W}_{\mathrm{ki}}^{\mathrm{h}+1} \\
& \mathrm{~S}_{\mathrm{i}}^{\mathrm{h}}=\sum_{\mathrm{j}} \mathrm{W}_{\mathrm{ij}}^{\mathrm{h}} \mathrm{O}_{\mathrm{j}}^{\mathrm{h}-1}
\end{aligned}
$$

where $f_{i}^{h}$ is the transfer functions which is usually the same for most neurons, and the boundary condition is:

$$
\mathrm{B}_{\mathrm{i}}^{\mathrm{L}}=\frac{\partial \varepsilon_{\mathrm{i}}}{\partial \mathrm{S}_{\mathrm{i}}^{\mathrm{h}}}=\mathrm{T}_{\mathrm{i}}-\mathrm{O}_{\mathrm{i}}^{\mathrm{L}}
$$

Thus in short the errors are propagated backwards in an essentially linear fashion using the transpose of the forward matrices, hence the symmetry of the weights, with a multiplication by the derivative of the corresponding forward activations every time a layer is traversed.

\section{Results}

In this section, we presents the results of brain functional connectivity detection using GICA on the migraine and HC fMRI datasets, as well as the classification performance evaluation results between these two groups obtained by BP combined with genetic algorithm.

Fig. 1 shows the 11 resting-state brain networks and their corresponding MNI co-ordinates obtained by analyzing the brain functional connectivity of migraine patients and healthy subjects at the group-level using GICA. These networks are subcortical networks (SCN), default mode networks (DMN), visual networks (VIN), lateral visual networks (LVN), auditory networks (AUN), salience networks (SAN), executive control networks (ECN), attention networks (ATTN), fronto-parietal networks (FPN), sensorimotor networks (SMN) and cognitive control networks (CCN), respectively, which are obtained with threshold $|z| \geq 2$ after z-scored the ICs of GICA。

Fig. 2 shows the parameter optimization results of BP neural network by genetic algorithm, and it can be found from the figure that the optimal optimization parameters are obtained when the algorithm iterates 74 times. Fig. 3 shows the ROC curves for the classification of migraine patients and healthy subjects by the BP algorithm using the parameters obtained in Fig. 2 on the training data, validation data and test data, as well as the overall performance, where the training data, validation data and test data are selected randomly from all sample data. It can be seen from the figure that the 
FCs between the 11 resting state brain networks (see in Fig. 1) can achieve 100\% accurate classification of migraine patients, which indicates that these resting state brain networks and corresponding FCs have a good identification performance for migraine patients in this paper.

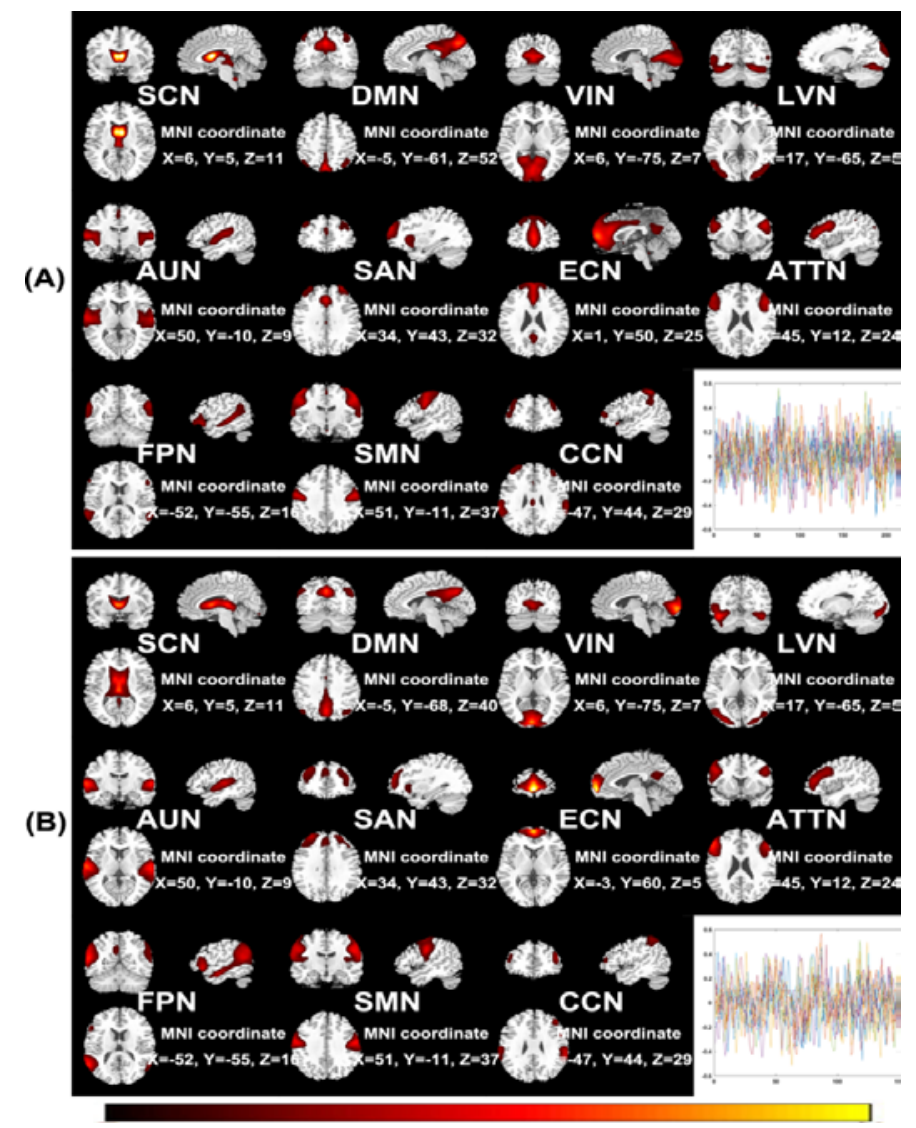

Fig.1 The 11 resting-state networks including SCN, DMN, VIN, LVN, AUN, SAN, ECN, ATTN, FPN, SMN and CCN as well as their corresponding MNI coordinates, in which (A) represents the healthy control group; (B) represents the migraine group. The last sub-graphs in (A) and (B) represent the corresponding time courses of the networks shown in the figure, respectively.

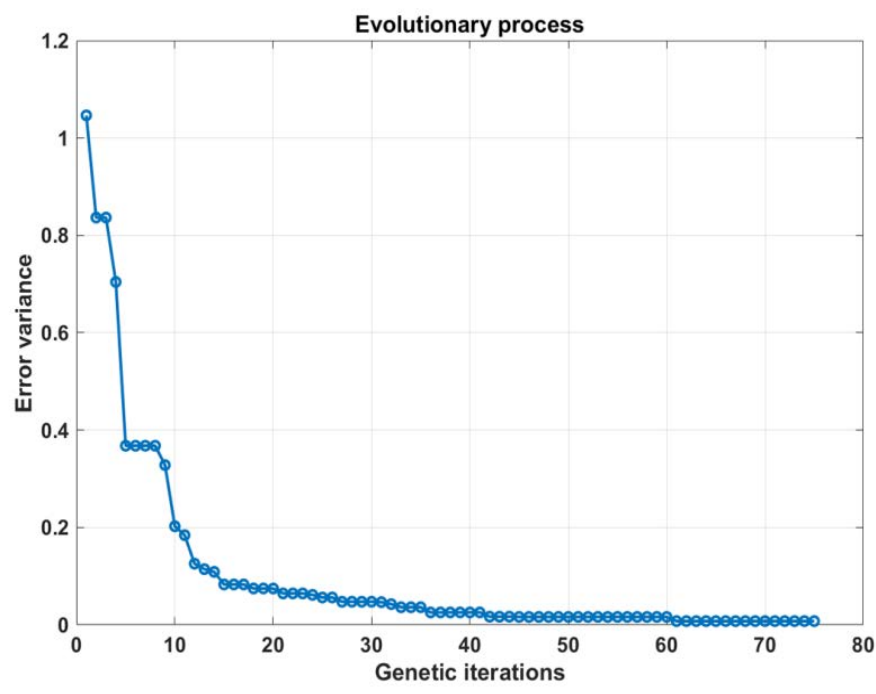

Fig.2 The iterations number corresponding error variance in the evolutionary process of BP parameters optimization by genetic algorithm. 

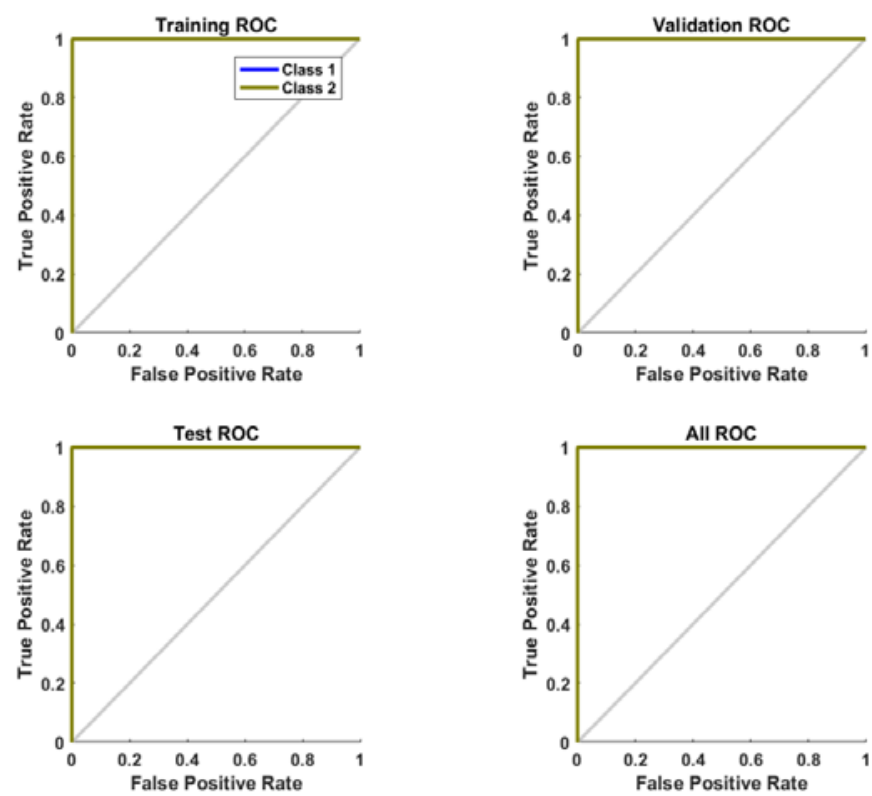

Fig.3 The ROCs of classification between migraine patients and HC subjects obtained using BP on the training data, validation data and test data, as well as the overall performance.

\section{Conclusion}

Identification of migraine using resting-state fMRI provides insights into pain circuits that are altered in migraine and could potentially contribute to the development of a new, noninvasive migraine biomarker. In this study, 11 classical resting-state brain functional networks were obtained from fMRI data of migraine patients and healthy subjects using GICA method, and then functional connectivity maps between these brain networks were constructed, which were used as features for the accurate classification of migraine patients and healthy subjects through genetic optimization and BP algorithm. This suggests that there was a significant difference between the brain functional connectivity characteristics of the 11 resting-state networks in migraine patients and healthy subjects, which means that they have been obvious changed in the brain function activities of migraine patients. The results may be provided a potential research perspective to reveal the pathogenesis of migraine and to form a new biomarker.

\section{Acknowledgments}

This work was sponsored by Shanghai Sailing Program (Grant No. 19YF1419000), and National Natural Science Foundation of China (Grant No. 61906117).

\section{References}

[1] E.A. Moulton, L. Becerra, N. Maleki, G. Pendse, S. Tully, R. Hargreaves, R. Burstein, and D. Borsook, "Painful heat reveals hyperexcitability of the temporal pole in interictal and ictal migraine states,” Cereb Cortex, vol. 21, pp. 435-448, 2012.

[2] N. Maleki, C. Linnman, J. Brawn, R. Burstein, and D. Borsook, "Her versus his migraine: Multiple sex differences in brain function and structure,” Brain, vol. 135, pp. 2546-2559, 2012.

[3] T.J. Schwedt, C.D. Chong, C.C. Chiang, L. Baxter, B.L. Schlaggar, Dodick, D.W.: Enhanced pain-induced activity of pain-processing regions in a case-control study of episodic migraine. Cephalalgia 34, 947-958 (2014). 
[4] T.J. Schwedt, B.L. Schlaggar, S. Mar, T. Nolan, R.S. Coalson, B. Nardos, T. Benzinger, and L.J. Larson-Prior, "Atypical restingstate functional connectivity of affective pain regions in chronic migraine,” Headache, vol. 53, pp. 737-751, 2013.

[5] V.A. Mathur, S.A. Khan, M.L. Keaser, C.S. Hubbard, M. Goyal, and D.A. Seminowicz, "Altered cognition-related brain activity and interactions with acute pain in migraine," Neuroimage Clin, vol. 7, pp. 347-358, 2015.

[6] A. Russo, A. Tessitore, F. Esposito, L. Marcuccio, A. Giordano, R. Conforti, A. Truini, A. Paccone, F. d'Onofrio, and G. Tedeschi, "Pain processing in patients with migraine: An event-related fMRI study during trigeminal nociceptive stimulation,” J Neurol, vol. 259, pp. 1903-1912, 2012.

[7] M.E. Raichle, “The restless brain,” Brain Connect, vol. 1, no. 1, pp. 3-12, 2011.

[8] A. Tessitore, A. Russo, F. Conte, A. Giordano, M. De Stefano, L. Lavorgna, D. Corbo, G. Caiazzo, F. Esposito, and G. Tedeschi, "Abnormal connectivity within executive resting-state network in migraine with aura,” Headache, vol. 55, pp. 794-805, 2015.

[9] C. Mainero, J. Boshyan, and N. Hadjikhani, “Altered functional magnetic resonance imaging resting-state connectivity in periaqueductal gray networks in migraine,” Ann Neurol, vol. 70, pp. 838-845, 2011.

[10] C. Jin, K. Yuan, L. Zhao, D. Yu, K.M. von Deneen, M. Zhang, W. Qin, W. Sun, and J. Tian, "Structural and functional abnormalities in migraine patients without aura," NMR Biomed, vol. 26, pp. 58-64, 2013.

[11] H. Ung, J.E. Brown, K.A. Johnson, J. Younger, J. Hush, and S. Mackey, "Multivariate classification of structural MRI data detects chronic low back pain,” Cereb Cortex, vol. 24, pp. 1037-1044, 2014.

[12] B. Sundermann, M. Burgmer, E. Pogatzki-Zahn, M. Gaubitz, C. Stüber, E. Wessolleck, G. Heuft, and B. Pfleiderer, "Diagnostic classification based on functional connectivity in chronic pain: Model optimization in fibromyalgia and rheumatoid arthritis,” Acad Radiol, vol. 21, pp. 369-377, 2014.

[13] C.D. Chong, N. Gaw, Y. Fu, J. Li, T. Wu, and T.J. Schwedt, "Migraine classification using magnetic resonance imaging resting-state functional connectivity data," Cephalalgia, vol. 37, no. 9, pp. 828-844, 2017.

[14] V.D. Calhoun, T. Adali, G.D. Pearlson, and J.J. Pekar, "A method for making group inferences from functional MRI data using independent component analysis,” Hum Brain Mapp, vol. 14, pp. 140-151, 2001.

[15] E.B. Erhardt, S. Rachakonda, E.J. Bedrick, E.A. Allen, T. Adali, and V.D. Calhoun, "Comparison of multi-subject ICA methods for analysis of fMRI data," Hum Brain Mapp, vol. 32, pp. 2075-2095, 2011.

[16] A. Hyvarinen, and E. Oja, “A fast fixed-point algorithm for independent component analysis,” Neural Comput, vol. 9, pp. 1483-1492, 1997.

[17] Y.O. Li, T. Restali, and V.D. Calhoun, "Estimating the number of independent components for functional magnetic resonance imaging data,” Hum Brain Mapp, vol. 28, pp. 1251-1266, 2007.

[18] J. Himberg, A. Hyvarinen, and F. Esposito, "Validating the independent components of neuroimaging time series via clustering and visualization,” Neuroimage, vol. 22, pp. 1214-1222, 2004. 
[19] H. Kwasnicka, and M. Przewozniczek, "Multi population pattern searching algorithm: a new evolutionary method based on the idea of messy genetic algorithm,: IEEE Trans Evol Comput, vol. 15, pp. 715-734, 2011.

[20] D.E. Rumelhart, G.E. Hinton, and R.J. Williams, "Learning internal representations by error propagation,” California Univ San Diego La Jolla Inst for Cognitive Science, Tech. Rep., 1985.

[21] A. Krizhevsky, I. Sutskever, G.E. Hinton, "Imagenet classification with deep convolutional neural networks,” In Advances in neural information processing systems, pp. 1097-1105, 2012.

[22] D. Silver, A. Huang, C.J. Maddison, A. Guez, L. Sifre, G. Van Den Driessche, J. Schrittwieser, I. Antonoglou, V. Panneershelvam, M. Lanctot, S. Dieleman, D. Grewe, J. Nham, N. Kalchbrenner, I. Sutskever, T. Lillicrap, M. Leach, K. Kavukcuoglu, T. Graepel, and D. Hassabis, "Mastering the game of go with deep neural networks and tree search,” Nature, vol. 529, no. 7587, pp. 484-489, 2016 